\title{
Two New and Four Unrecorded Species of Chironomidae (Diptera) in Korea
}

\author{
Han-Il Ree ${ }^{1, *}$, Sung Hyun Nam ${ }^{1}$, Kyoung Yong Jeong ${ }^{2}$ \\ ${ }^{1}$ Department of Environmental Medical Biology and Institute of Tropical Medicine, \\ College of Medicine, Yonsei University, Seoul 120-752, Korea \\ ${ }^{2}$ Department of Internal Medicine and Institute of Allergy, \\ Korea National Arthropods of Medical Importance Resource Bank, College of Medicine, \\ Yonsei University, Seoul 120-752, Korea
}

\begin{abstract}
Chironomid adults attracted to the light were collected at Dangsan-ri, Muju-eup, Muju-gun, Jeollabuk-do in 2008-2009. Two new species, Lymnophyes parakitanaides sp. nov. and Parakiefferiella mujuensis sp. nov., and four unrecorded species, Cardiolcladius capusinus, Thienemanniella vittata, Conchapelopia pallidula, and Nilotanypus dubius were found and are described with illustrations. The genera Thienemanniella and Nilotanypus have not been previously reported in Korea. The genus Thienemanniella which belongs to Orthocladiinae is characterized by the radial sector retracted and apically fused with the costa, and the genus Nilotanypus which belongs to Tanypodinae is characterized by the absence of $\mathrm{R}_{2+3}$, pubescent eyes and lack of the gonocoxal lobes.
\end{abstract}

Keywords: new species, Chironomidae, Muju-gun, Korea, taxonomy

\section{INTRODUCTION}

Non-biting midges (Diptera, Chironomidae) include a large number of species, whose larvae are found in almost all types of waters. Emerged in large numbers and attracted to electric lights, they cause unpleasant living and play an important role in allergic diseases (Lee et al., 1995; Yong et al., 1999).

Total 1,113 adults were collected near Namdae Stream located in Muju-gun in 2008-2009. One new and eight newly recorded species of the genus Polypedilum were reported by Ree et al. (2010), and six new and four unrecorded species of tribe Tanytarsisni were also described by Ree et al. (2011). In addition, we report two new and four unrecorded species. The genera Thienemanniella and Nilotanypus are the first record in Korea. The type specimens were deposited in the collection of Arthropods of Medical Importance Resource Bank, Department of Environmental Medical Biology, Yonsei University. Therminology followed Saether (1980).

\section{MATERIALS AND METHODS}

Adult chironomid collections were carried out near Namdae

(c) This is an Open Access article distributed under the terms of the Creative Commons Attribution Non-Commercial License (http://creativecommons.org/ licenses/by-nc/3.0/) which permits unrestricted non-commercial use, distribution, and reproduction in any medium, provided the original work is properly cited.

pISSN 2234-6953
Stream located in Dangsan-ri, Muju-eup, Muju-gun, Jeollabuk-do on September 2008 and in May and August 2009. Adults attracted to light sources of various buildings and advertising signage of restaurants and stores. Namdae Stream runs through the western area of Muju-gun, and joins to the Keum River. The many restaurants are located along the stream in the vicinity of the $120 \mathrm{~mm}$ long Namdaecheon Bridge. Large numbers of non-biting midges and mayflies arise from the stream.

Aspirated adults were preserved in $75 \%$ ethanol, and later they were dissected under stereomicroscope using fine needles to retrieve the antennae, head, wings, abdomen and hypopygium, and were mounted on polyvinyl alcohol medium.

\section{SYSTEMATIC ACCOUNTS}

\author{
Order Diptera \\ Family Chironomidae Macquart \\ Subfamily Orthocladiinae Edwards \\ Genus Caradiocladius Kieffer
}

*To whom correspondence should be addressed

Tel: 82-2-2228-1840, Fax: 82-2-363-8676

E-mail: para@yuhs.ac 
1*Cardiocladius capusinus (Zetterstedt, 1850) (Fig. 1) Chironomus capusinus Zetterstedt, 1850: 3488.

Cardiocladius capusinus: Edwards, 1929: 317; Goetghebuer, 1932: 48; Tokunaga, 1939: 308; Brundin, 1956: 66; Pinder, 1978: 56; Sasa and Okazawa, 1992: 66.

Material examined. $1 \sigma^{7}$ (R-CH-6448), Korea: Jeollabuk-do, Muju-gun, Muju-eup, Dangsan-ri, 5 Sep 2008, Jeong KY, Nam SH.

Diagnosis. Small to medium (wing length [WL] $1.5 \mathrm{~mm}$ ), yellowish brown species. Anal tergite with two lobes located posteriorly at middle. Inner lobe of gonocoxite long, finger-like, with numerous setae. Abdominal tergite brownish yellow, with a large and rectangular dark patch. Antennal ratio (AR) 1.31. Leg ratio (LR) 0.68 .

Description (male). Head: Eye bare, not dorsomedially produced. Antenna brown, with 13 segments; AR 1.31. Clypeus yellowish brown, with about 50 setae. Palp pale dark brown, with five segments: $36,52,114,179,173 \mu \mathrm{m}(1: 1.5: 3.2$ : $5.0: 5.4)$; 3rd segment unusually wider $(114 \mu \mathrm{m}$ long $\times 68$ $\mu \mathrm{m}$ wide) and many setae (Fig. 1C). Thorax: Yellowishbrown in ground color. Antepronotum well-developed, yellowish-brown. Scutum yellowish-brown, with vandike-brown vittae; about 20 minute, decumbent acrosticals; 17-19 erect dorsocentrals, arising from pale pits and 9 prealars each side. Scutellum brown, with about 30 setae. Postnotum dark brown. Halter pale yellow. Wing (Fig. 1A): WL $1.5 \mathrm{~mm}$. Membrane bare. Costa not produced. $\mathrm{R}_{2+3}$ end near $\mathrm{R}_{1} \cdot \mathrm{R}_{4+5}$ distal to $\mathrm{M}_{3+4}$. RM under $\mathrm{FCu}$. $\mathrm{Cu}_{1}$ almost straight. Anal lobe developed. Squama fringed. Brachiolum and arculus dark brown. Legs: Uniformly brown. Fore tibia with a sharp apical spur; mid tibia with 2 strong spurs, mid tarsi I-III with 2 short spurs; hind tibia with 2 strong spurs and several weak spurs, hind tarsi I-III with 2 short spurs. Tarsus IV shorter than tarsus V, and bilobed at tip (Fig. 1D). Pulvillus absent. LR 0.68. Abdomen: All tergite brownish-yellow, each with a large rectangular brown patch and numerous setae arising from small, pale pits. Hypopygium (Fig. 1B): Anal tergite with 2 lobes posteriorly at middle, without median and apical setae. Anal point absent. Inner lobe of gonocoxite rather long, finger-like, with numerous setae. Gonostylus with smoothly rounded tip, a ventrally directed megaseta.

Remarks. Cardiocladius capusinus is very similar to $C a$. fuscus in morphological characters. The main difference is shorter tarsus IV in capusinus. The ratio of tarsus IV/V was 0.74 in capusinus and 0.79 in fuscus (Pinder, 1978). The ratio of tarsus IV/V of Korean fuscus (R-CH-1094) collected in Samcheok, Gangwon-do in 1979 is 0.64 (Ree and Kim, 1981) and that of our capusinus species was 0.60 .

\section{Genus Lymnophyes Eaton}

\section{${ }^{2 *}$ Lymnophyes parakitanaides $\mathbf{s p . ~ n o v . ~}$}

Material examined. Holotype, $1 \sigma^{7}$ (R-CH-6452), Korea: Jeollabuk-do, Muju-gun, Muju-eup, Dangsan-ri, 5 Sep 2008, Jeong KY, Nam SH. Paratype, 5 ठ ণ ס (R-Ch-6450, 6761, 6961, 6967, 6984), data as same as holotype.

Diagnosis. Dark brown to yellow-brown, small midges (WL $1.02 \mathrm{~mm}$ ). 1-3 setae on each of epimeron II, preepisternum and posterior anepisternum II; lamellar setae on scutum absent. Anal tergite prominentely produced posteriorly, with a pair of rather small lobes on middle. Gonocoxite large, with smoothly rounded inner lobe. AR 0.64. LR 0.56.

Description (male). Head: Eye reniform, bare. Two postoculars. Antenna dark brown, with 13 segments. AR 0.64. Palp pale dark brown, with 5 segments: $20,23,56,46,57 \mu \mathrm{m}$ (1 : $1.2: 2.8: 2.3: 2.9$ ). Clypeus brown, with 15 setae, uniserial. Thorax: Dark brown in ground color. Antepronotum dark brown, moderately developed, lobe just separated, with 1 dorsal seta and 1 ventral seta. Scutum brown, vittae not distinguishable; 8-9 long, erect dorsocentrals and 6-7 prealars each side. Scutellum dark brown, with 9 setae. Postnotum dark brown. 3 setae on epimeron II, 3 setae on preepisternum, and 1-2 posterior anepisterum II. Halter dark brown at tip. Wing (Fig. 2A): WL $1.02 \mathrm{~mm}$. Membrane bare, coarsely dotted with microtrichiae. Costa well produced. $\mathrm{R}_{2+3}$ ending at middle between $\mathrm{R}_{1}$ and $\mathrm{R}_{4+5}$. FCu distal to $\mathrm{RM}$. $\mathrm{Cu}_{1}$ bent at middle. Anal lobe not developed. Squama with 4 setae. Arculus pale, brachiolum pale with 1 seta. Legs: Uniformly yellowish brown. Fore tibia with a long apical spur; mid tibia with 2 spurs; hind tibia with 2 spurs and several comb spurs. Pulvillus absent. LR 0.56. Abdomen: Tergite I-IV pale brown and V-VII yellowish dark brown. Hypopygium (Fig. 2B): Anal tergite smoothly rounded distally, with a pair of dark brown lobes at middle. Gonocoxite large, with smoothly rounded inner lobe. Gonostylus parallel-sided, with a prominent megaseta and a smaller pseudospur at tip.

Etymology. The name Lymnophyes parakitanaides is given for its similar morphological appearance with L. tamakitanaides.

Remarks. This new species is similar to L. tamakitanaides described by Sasa (1981), but differs in the following characters: (1) body coloration of the former is dark brown, with yellowish-brown legs and pale brown abdominal tergite I$\mathrm{IV}$, whereas the later species is almost completely dark brown including legs and abdomen; (2) a pair of the lobe of anal tergite in the former is rather small without setae and located in the middle, whereas those of the later is larger, with

Korean name: ${ }^{1 *}$ 황갈마디깔따구(신칭), ${ }^{2 *}$ 아기 볼물깔따구(신칭) 


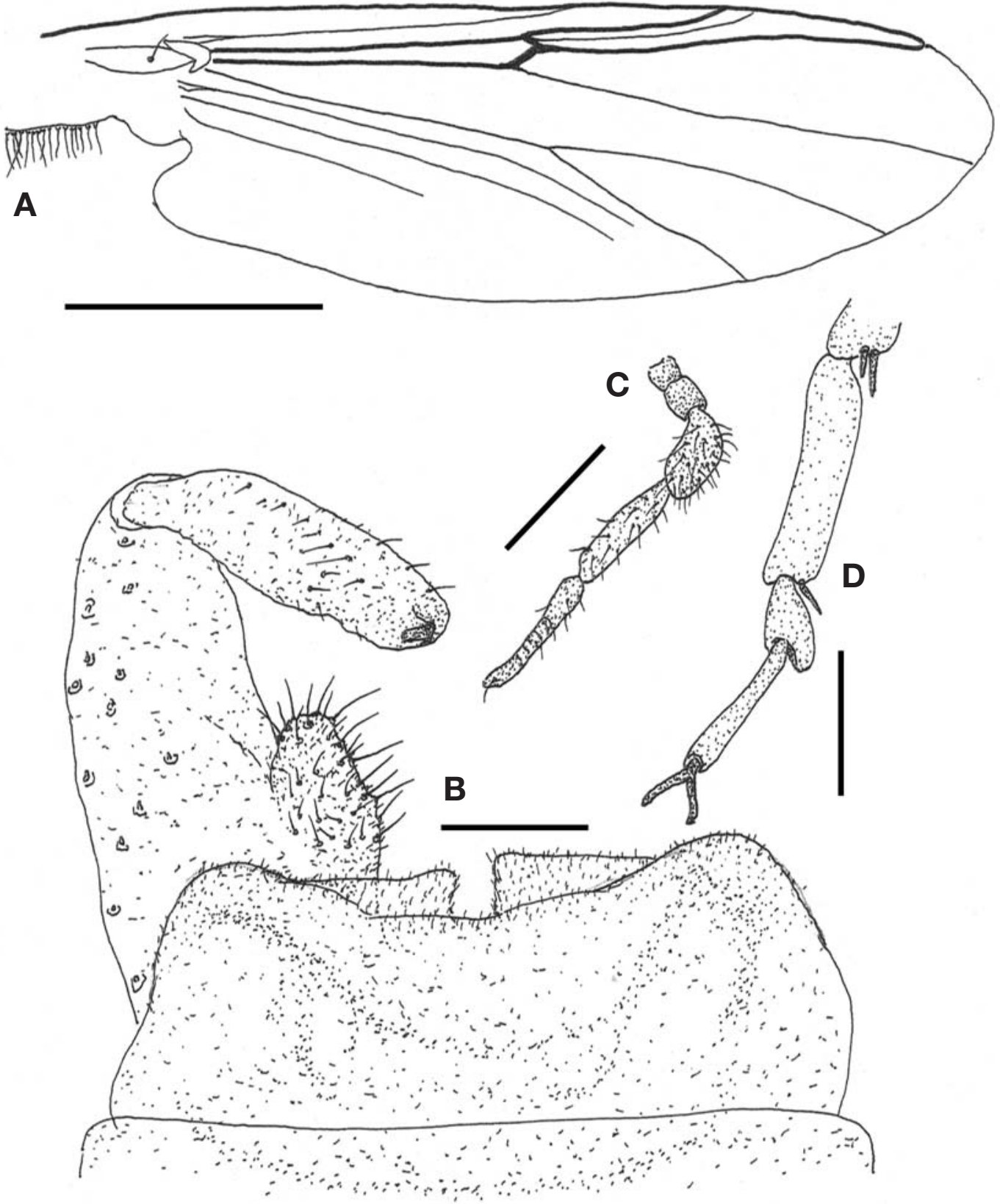

Fig. 1. Cardiocladius capusinus (male). A, Wing; $B$, Hypopygium; $C$, Palp; $D, 3$ rd-5th tarsi of hind leg. Scale bars: $A=0.5$ mm, $\mathrm{B}=0.05 \mathrm{~mm}, \mathrm{C}=0.2 \mathrm{~mm}, \mathrm{D}=0.1 \mathrm{~mm}$.

several setae, and located on posterior margin of the tergite.

Genus Parakiefferiella Thienemann

1*Parakiefferiella mujuensis sp. nov.

Material examined. Holotype, $1 \sigma^{\top}$ (R-CH-6945), Korea:
Jeollabuk-do, Muju-gun, Muju-eup, Dangsan-ri, 22 May 2009, Jeong KY. Paratype, $2 \sigma^{\nearrow}$ 『 (R-CH-6451, 6564), locality same as holotype, 15 Sep 2008, Jeong KY, Nam SH; 2 ఠ శ (R-CH6834, 6872), same as holotype.

Diagnosis. Brownish-yellow, small to medium midges (WL $1.45 \mathrm{~mm}$ ). Anal point rather short, pale, with smoothly rounded tip. Gonocoxite long, rather slender, with 2 inner lobes:

Korean name: ${ }^{1 *}$ 무주숲깔따구 (신칭) 

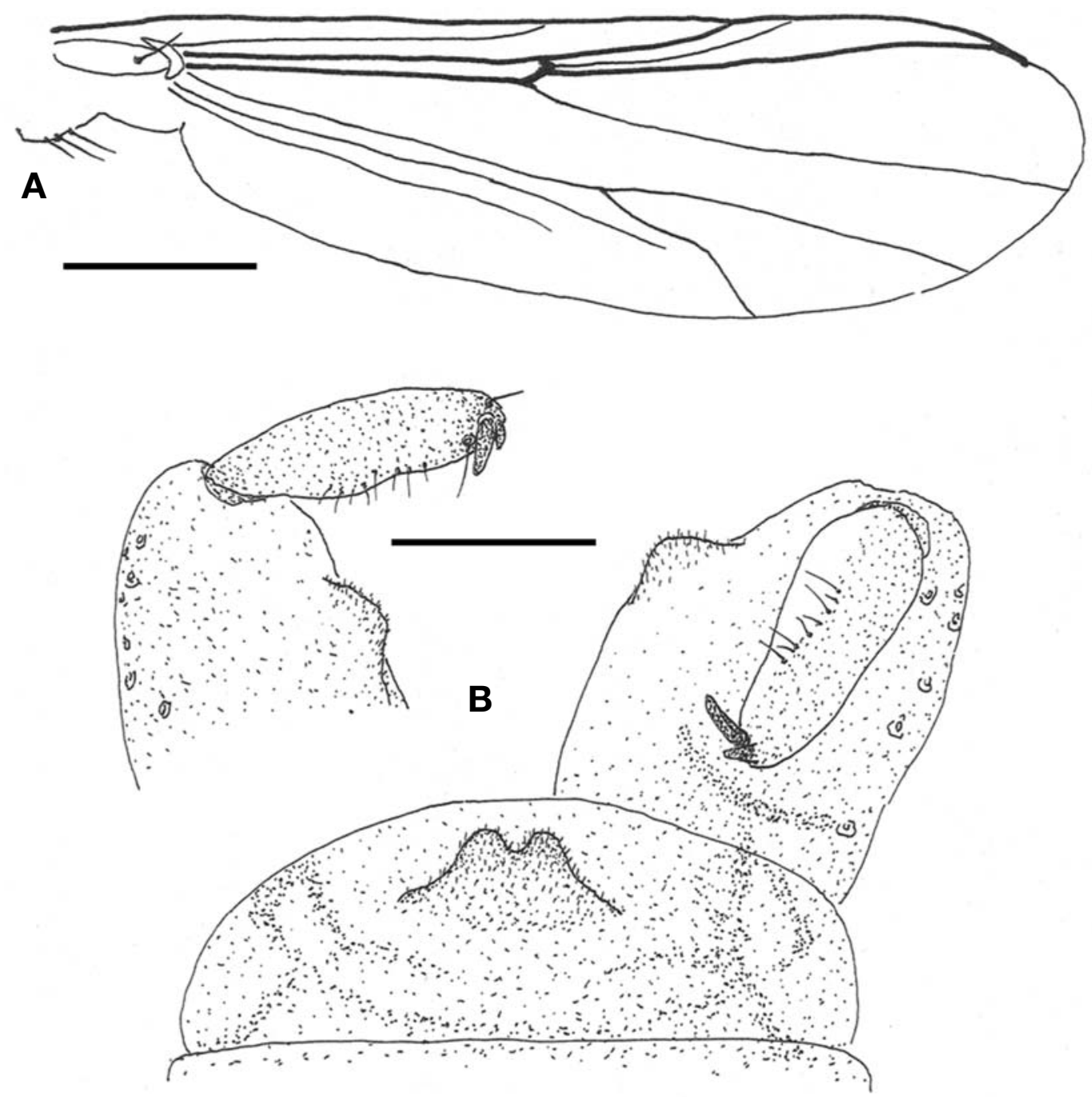

Fig. 2. Lymnophyes parakitanaides sp. nov. (male). A, Wing; B, Hypopygium. Scale bars: $A=0.2 \mathrm{~mm}, \mathrm{~B}=0.05 \mathrm{~mm}$.

dorsal one pale, bare, ventral one covered with microtrichia and short setae. AR 0.98. LR 0.54.

Description (male). Head: Eye reniform, bare. Antenna pale dark brown, with 13 segments. AR 0.98. Palp pale, with 5 segments: $29,32,71,102,171 \mu \mathrm{m}(1: 1.1: 2.5: 3.5: 5.9)$. Clypeus brownish-yellow, with 10 setae. Thorax: Brownishyellow in ground color. Antepronotum brownish-yellow, narrowed dorsally. Scutum brownish-yellow, with dark brown vittae; acrosticals absent. Scutellum brownish-yellow, with 2 setae. Postnotum dark brown. Wing (Fig. 3A): WL $1.45 \mathrm{~mm}$. Membrane bare, transparent. Costa well produced. $\mathrm{R}_{2+3}$ runnning close to $\mathrm{R}_{4+5}$. FCu distal to $\mathrm{RM}$. $\mathrm{Cu}_{1}$ bent at middle. An reaching beyond $\mathrm{FCu}$. Anal lobe moderately developed. Squama bare. Arculus pale, brachiolum pale with 1 seta. Legs: All segments uniformly pale yellow. Fore tibia with an apical spur; mid tibia with 2 short, pale spurs; hind tibia with a long spur and 9-11 free comb spurs. Pulvullus absent. LR 0.54. Abdomen: All segments uniformly pale yellow. Hypopygium (Fig. 3B): Anal tergite hemisphere distally, with 10-11 median setae. Apodemes pale, not well developed (not clearly seen). Anal point pale, rather short, with smoothly rounded tip. Gonocoxite long, rather slender, with 2 gonocoxite inner lobes: dorsal one pale and bare, ventral one covered with microtrichia and many short setae (Fig. 3C). Gonostylus slender, parallel-sided, with a pale, megaseta and 2 subapical setae.

Etymology. The new name refers to the place where this species was first collected.

Remarks. Two forms are observed in color of scutal vittae; one dark brown and the other brownish yellow which is not clearly distinguishable. Double lobes of gonocoxite of this new species are similar to those of Orthocladius suspensus, 

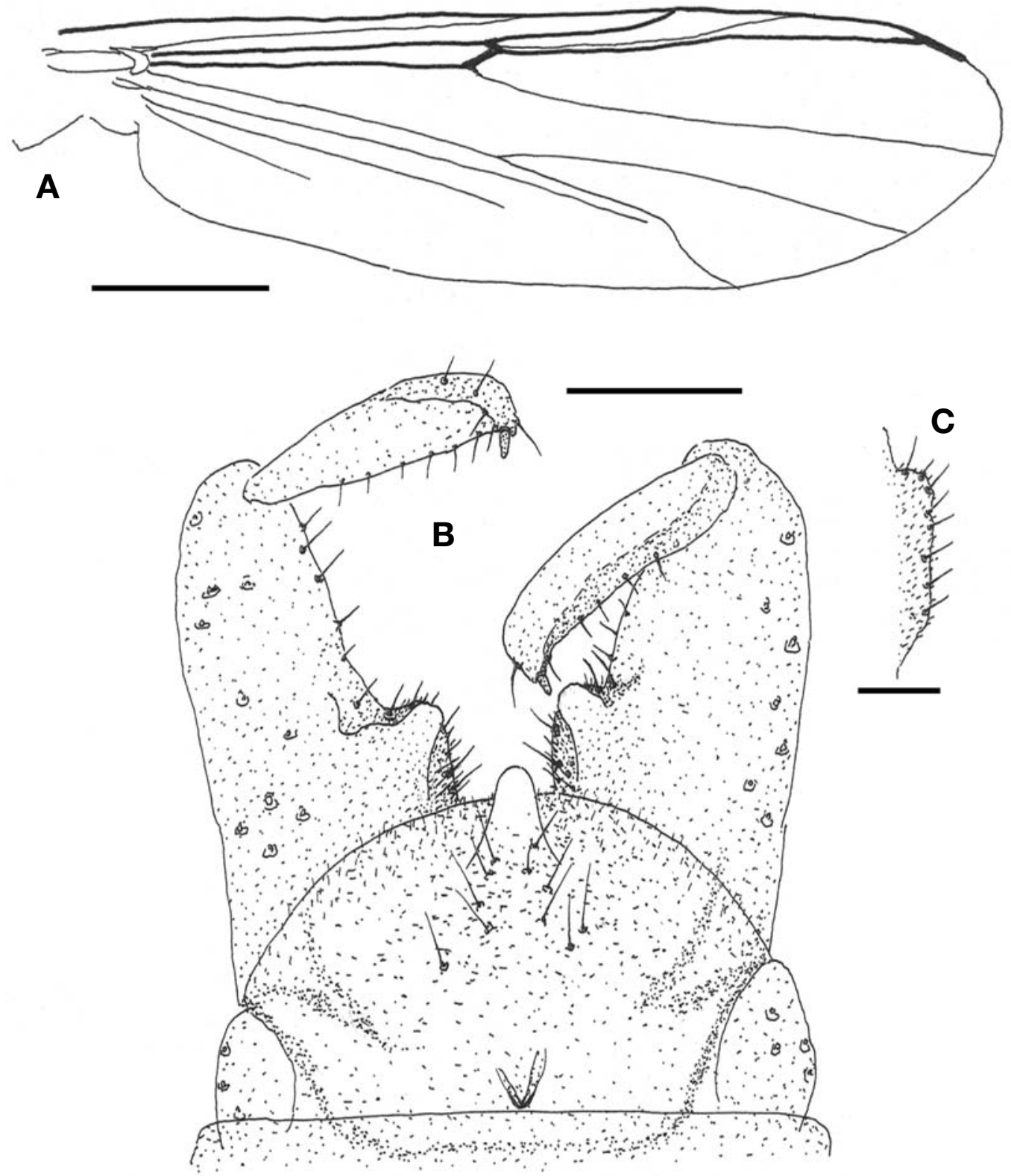

Fig. 3. Parakiefferiella mujuensis sp. nov. (male). A, Wing; B, Hypopygium; C, Ventral lobe of gonocoxite. Scale bars: A=0.3 mm, $B=0.05 \mathrm{~mm}, C=0.03 \mathrm{~mm}$.

but differs in the ventral lobe, which is rectangular in the former, whereas triangular in the later (Ree and Kim, 1981).

\section{${ }^{1 *}$ Genus Thienemanniella Kieffer}

${ }^{2 *}$ Thienemanniella vittata (Edwards, 1924)

Corynoneura (Thienemanniella) vittata Edwards, 1924: 182. Thienemanniella vittata: Edwards, 1929: 366; Pinder, 1978:
98; Sasa and Kawai, 1987: 54; Sasa, 1990: 49.

Material examined. 5 ऽ ๙ , Korea: Jeollabuk-do, Muju-gun, Muju-eup, Dangsan-ri, 22 May 2009, Jeong KY. 5 万্ locality and collector same, 28 Aug 2009.

Diagnosis. Brownish-yellow, small midges (WL $0.98 \mathrm{~mm}$ ). Antenna pale brown, with 12 segments. Costa fused with $R_{1}$, forming thick clavus, ending about $1 / 3$ of wing length.

Korean name: ${ }^{1 *}$ 몽당깔따구속(신칭), ${ }^{2 *}$ 좀몽당깔따구(신칭) 

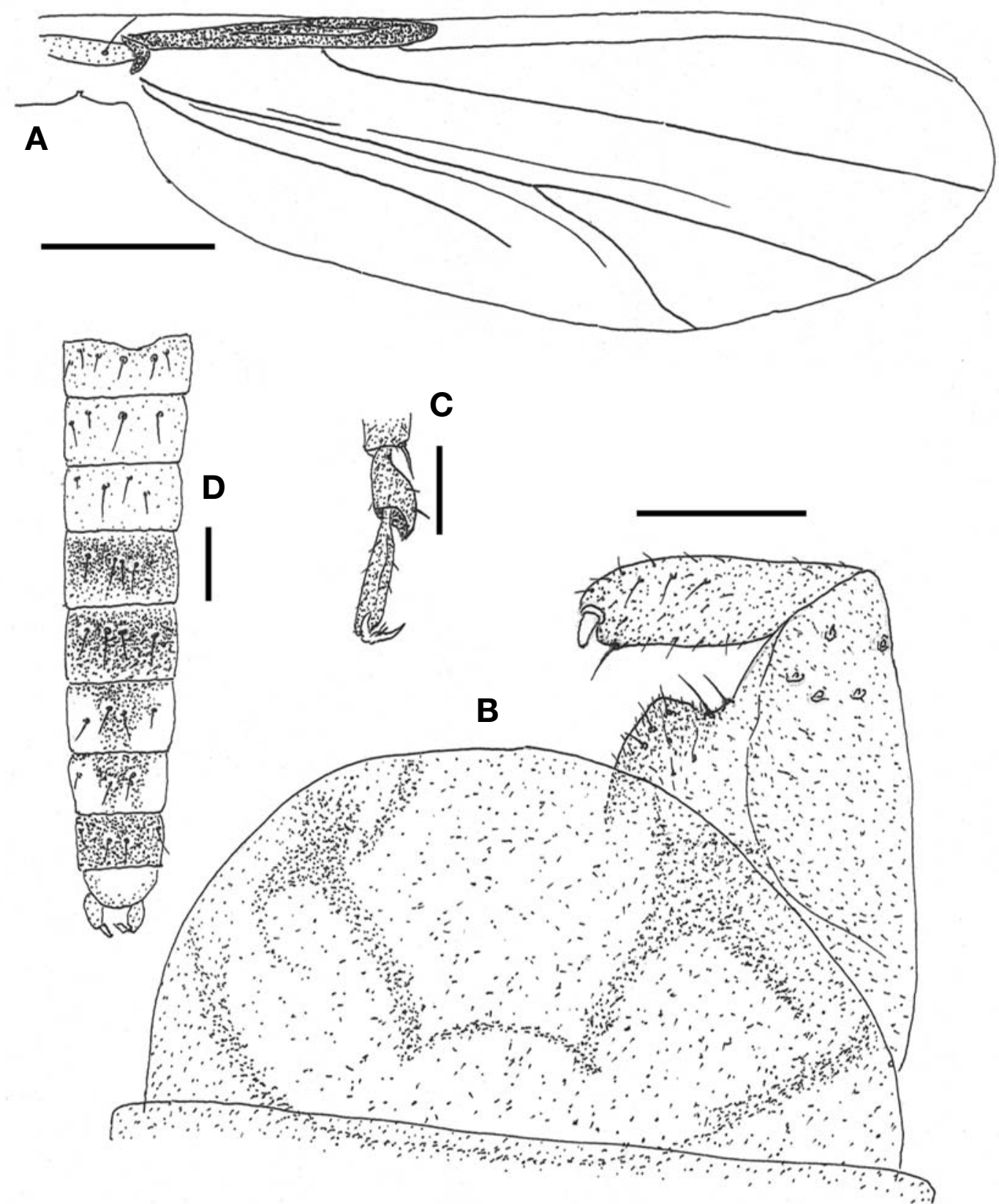

Fig. 4. Thienemanniella vittata (male). A, Wing; $B$, Hypopygium; $C, 4$ th and 5 th segments of hind tarsi; $D$, Andomen (dorsal). Scale bars: $A=0.2 \mathrm{~mm}, B, C=0.03 \mathrm{~mm}, D=0.1 \mathrm{~mm}$.

$\mathrm{R}_{4+5}$ not fused with $\mathrm{R}_{1}$, reaching apex of wing. Anal point $\mathrm{ab}-$ sent. Inner lobe of gonocoxite somewhat triangular, covered with microtrichia. AR 0.58. LR 0.91.

Description (male). Head: Eye reniform, pubescent. Postorbitals absent. Clypeus narrow, with 10 setae arranged biserially. Antenna pale brown, with 12 segments. AR 0.58. Palp pale, with 4 segments: $14,41,54,99 \mu \mathrm{m}(1: 2.9: 3.9: 7.1)$. Thorax: Brownish-yellow in ground color. Antepronotum brownish-yellow, narrowed dorsally, widely separated. Scu- tum brownish-yellow, with dark brown vittae; acrosticals absent, 7 dorsocentrals and 3 prealars each side. Scutellum dark brown, with 2 setae. Postnotum dark brown. Wing (Fig. 4A): WL $0.98 \mathrm{~mm}$. Membrane bare, transparent. Costa fused with $R_{1}$, forming thick clavus, ending about $1 / 3$ of wing length. $\mathrm{R}_{4+5}$ not fused with $\mathrm{R}_{1}$, reaching apex of wing. $\mathrm{Cu}_{1}$ slightly bent. FCu far distal to RM. Anal lobe poorly developed. Squama bare. All veins bare. Arculus dark brown, brachiolum pale with 1 seta. Legs: All segments uniformly pale. 

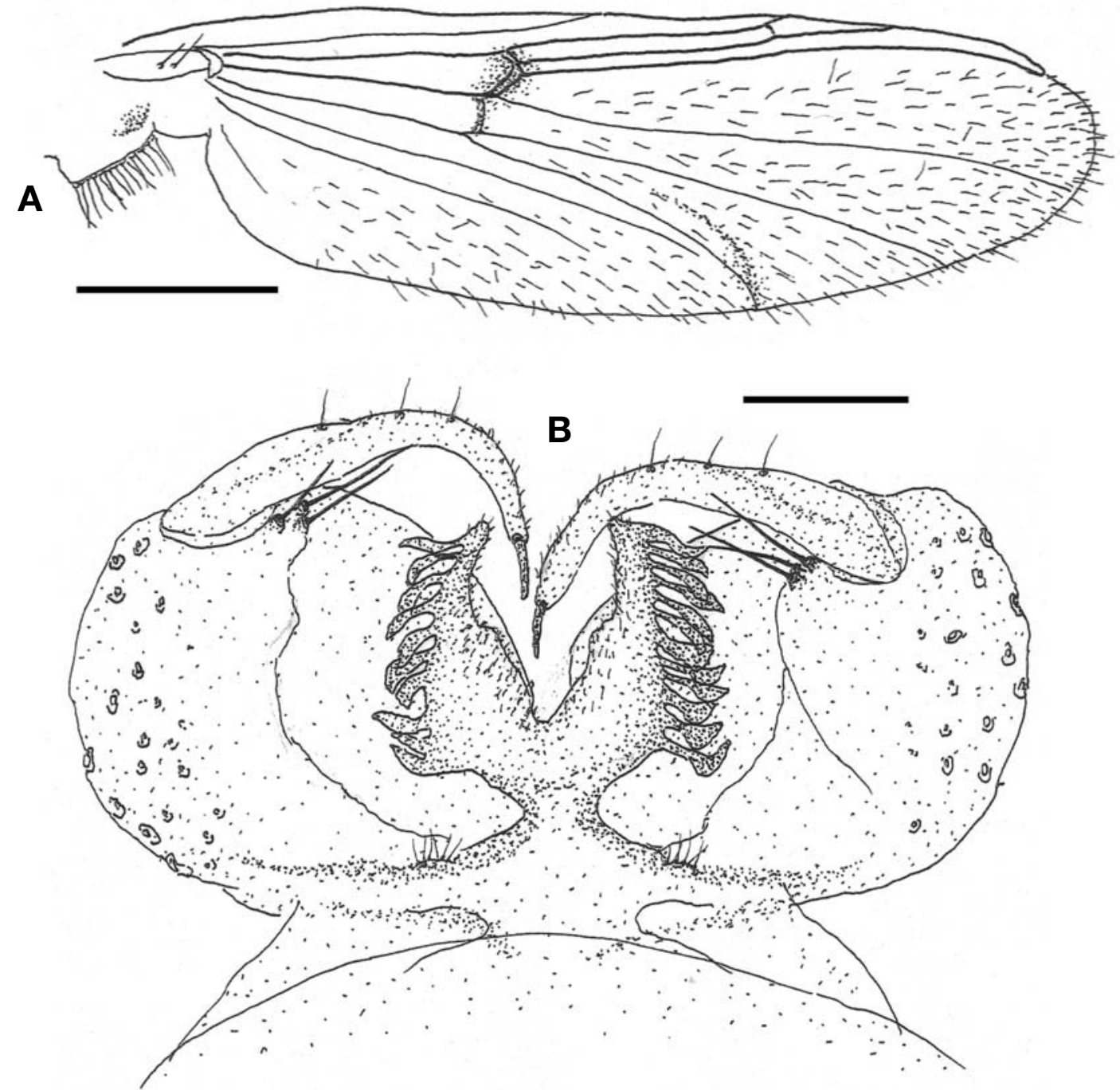

Fig. 5. Conchapelopia pallidula (male). A, Wing; $B$, Hypopygium. Scale bars: $A=0.2 \mathrm{~mm}, B=0.05 \mathrm{~mm}$.

Fore tibia with a long apical spur; mid tibia with 2 small spurs; hind tibia with 2 spurs and several comb spurs. Tarsus IV somewhat cardiform and shorter than tarsus V (Fig. 4C). Pulvillus absent. LR 0.91. Abdomen (Fig. 4D): Tergite I-III brownish pale yellow; tergite IV, V, VIII dark brown; tergite VI, VII pale with dark brown patch on middle. Hypopygium (Fig. 4B): Anal tergite comparatively large, hemisphere distally; apodemes strongly sclerotized. Anal point absent. Inner lobe of gonocoxite somewhat triangular, covered with microtrichia and several small setae. Gonostylus parallel-sided with a terminal spur.

Remarks. The genus Thienemanniella is the first record in Korea. This genus has a very unique characteristic that the radial sector of the wing is retracted, swollen and apically fused with the costa in a thick clavus, ending before the midpoint of the wing (Cranston et al., 1989). Most characters of our specimens are identical to those of Th. vittata described by Sasa (1990), except body coloration: ground color of thorax brownish-yellow and abdominal tergite I-III pale brownish-yellow in the former species, whereas ground color of thorax and abdominal tergite I-III brown in the latter.

Subfamily Tanypodinae Thienemann and Zavrel ${ }^{1 *}$ Genus Conchapelopia, Fittkau

2*Conchapelopia pallidula (Meigen, 1818)

Tanypus pallidus Meigen, 1818: 16.

Ablabesmyia pallidula: Goetghebuer, 1937: 36. 

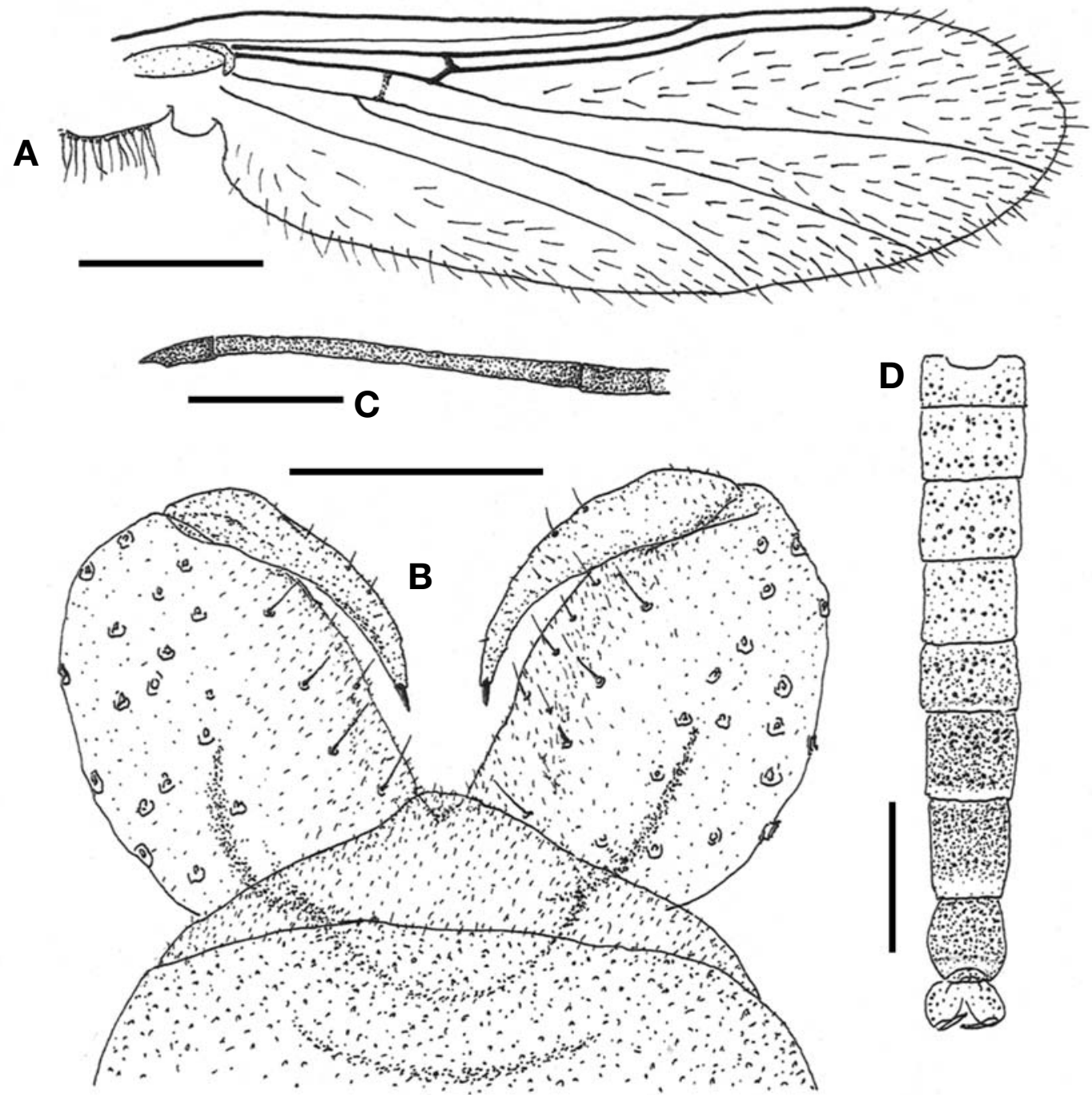

Fig. 6. Nilotanypus dubius (male). A, Wing; B, Hypopygium; C, Last 3 segments of antenna; D, Abdomen (dorsal). Scale bars: A, $D=0.2 \mathrm{~mm}, B=0.05 \mathrm{~mm}, C=0.1 \mathrm{~mm}$.

Conchapelopia pallidula: Fittkau, 1962: 236; Pinder, 1978: 32.

Material examined. $2 \sigma^{\nearrow}$ 『 (R-CH-6546, 6596), Korea: Jeollabuk-do, Muju-gun, Muju-eup, Dangsan-ri, 22 May 2009, Jeong KY.

Diagnosis. Pale yellow, medium-sized species (WL $2.2 \mathrm{~mm}$ ). Wing membrane densely covered with macrotrichia, without markings except around RM. MCu distal to FCu. Anal tergite pale, very small in size. Gonocoxite large, mussel-shell in form, with 3 strong setae on proximal base of gonostylus. Median volsella well developed, with a row of spoon shaped processes outerlaterally. Gonostylus long, slender, curved at middle. AR 1.54. LR 0.82.

Description (male). Head: Redish yellow in ground color.
Eye bare, dorsomedially extended. $12-13$ postoculars. Antenna pale brown, with 14 segments; last segments much shorter than penultimate one. AR (last 2 segments/1-12 segments) 1.54. Clypeus brownish yellow, oval in shape, with about 30 setae. Palp pale, with 4 segments: $112,166,216,288 \mu \mathrm{m}(1$ : $1.5: 1.9: 2.6)$. Thorax: Pale yellow in ground color. Antepronotum pale, narrowed dorsally, lobe separated. Scutum pale yellow, anterior half of dorsocentral and lateral vittae dark brown. Scutellum pale, with 35-40 setae. Postnotum brownish yellow. Wing (Fig. 5A): WL $2.2 \mathrm{~mm}$. Membrane densely covered with macrotrichia; Only area of RM slightly darker (brownish yellow), no other dark markings. Costa not produced. $\mathrm{R}_{2+3}$ forked. $\mathrm{R}_{4+5}$ ending distal to $\mathrm{M}_{3+4}$. $\mathrm{MCu}$ just distal to $\mathrm{FCu}$. An ending beyond $\mathrm{FCu}$. Anal lobe moderately developed. Squama with many setae. Arculus pale, brachi- 
olum pale with 2 setae. Legs: All segments uniformly pale yellow. Fore tibia with a short, dark spur; mid tibia with 2 spurs; hind tibia with a long spur, a short spur and free comb 7-8 free spurs. Pulvillus absent. LR 0.82. Abdomen: Tergite I-VI pale yellow, VII-VIII brown. Hypopygium (Fig. 5B): Anal tergite pale, extremely small. Gonocoxite large, somewhat flattened and round (mussel-shell in form), with 3 strong setae just proximal to basal portion of gonostylus. Median volsella well developed, with a row of spoon shaped processes outerlaterally. Gonostylus long, slender, curved at middle, with a dark brown megaseta.

Remarks. The most prominent character of this species is the spoon-shaped processes of median volsella along outerlateral margin. Pinder (1978) described that the processes of gonocoxite lobe are expanded subapically so that they look like somewhat spoon-like in shape, whereas those of our specimens look like more distinct spoon-like in shape.

\section{$1 *$ Genus Nilotanypus Kieffer}

\section{${ }^{2 *}$ Nilotanypus dubius (Meigen, 1818)}

Chironomus dibius Meigen, 1818: 11.

Ablabesmyia dubia: Goetghebuer, 1937: 40.

Nilotanypus dubius: Fittkau, 1962: 412; Pinder, 1978: 33.

Material examined. $3 \sigma^{7} \sigma^{\nearrow}$, Korea: Jeollabuk-do, Muju-gun, Muju-eup, Dangsan-ri, 28 Aug 2009, Jeong KY.

Diagnosis. Dark brown, rather small midges (WL $1.1 \mathrm{~mm}$ ). Eye hairy, with well developed dorsomedial extension. Wing membrane thickly covered with macrotrichia. Costa relatively short (about $3 / 4$ of wing length). $\mathrm{R}_{2+3}$ absent. $\mathrm{RM}$ at basal $1 / 4$ of wing length. $\mathrm{MCu}$ proximal to $\mathrm{RM}$. Anal tergite small, without any setae. Gonocoxite large, oval in shape, without inner lobe. AR 0.65. LR 0.78.

Description (male). Head: Eye hairy, dorsomedial projection well developed. Antenna dark brown, with 14 segments: last segment short (44.6 $\mu \mathrm{m})$ (Fig. 6C). AR (13-14 segments/ 1-12 segments) 0.65 . Palp pale yellow, with 4 segments: 29 , $76,108,90 \mu \mathrm{m}(1: 2.6: 3.7: 3.1)$. Clypeus brownish yellow, with 16 segments. Thorax: Dark brown in ground color. Antepronotum dark brown, sharply narrowed dorsally, with 5-6 setae ventrally. Scutum dark brown, vittae not clearly defined, 15-16 dorsocentrals and 9 prealars each side. Scutellum dark brown, with 8 long and many short scutellars. Postnotum dark brown. Wing (Fig. 6A): WL $1.1 \mathrm{~mm}$. Membrane thickly covered with macrotrichia. Costa relatively short (about 3/4 of wing length), not produced. $\mathrm{R}_{2+3}$ absent. RM located at about basal $1 / 4$ of wing length. $\mathrm{MCu}$ proximal to $\mathrm{RM}$, and distal to $\mathrm{FCu}$. $\mathrm{R}_{4+5}$ proximal to $\mathrm{M}_{3+4} \cdot \mathrm{Cu}_{1}$ long, almost straight. Anal lobe moderately developed. Squama with many setae. Arculus dark brown, brachiolum pale dark brown with 2 setae. Legs: Femurs pale dark brown. Tibiae pale yellow (fore tibia darker). All tarsi pale. Fore and mid tibiae with a long, pale spur; hind tibia with a long spur and 5 free comb spurs. Pulvillus absent. LR 0.78. Abdomen (Fig. 6D): Tergite I-IV pale dark brown; tergite V-VIII dark brown (VI with narrow distal pale band, and VII with wider distal pale band). Hypopygium (Fig. 6B): Tergite IX (anal tergite) very small, without any setae. Phallapodeme strong, most part located in gonocoxite. Transverse sternapodeme strong. Gonocoxite large, oval in shape, without inner lobe. Gonostylus narrow, sharply tapered apically, slightly bent, with a rather small megaseta.

Remarks. This is the frist description of the genus Nilotanypus in Korea. This genus has several unique characters that are separable from other genera of Tanypodinae: basal lobe of gonocoxite is absent, eyes are punescent, wings are unmarked, and $\mathrm{R}_{2+3}$ is absent. Most characteristics of our specimens generally fit to those of $\mathrm{Ni}$. dibius (Meigen), except the value of LR (0.78 in Korean specimens vs. 1.3 in European specimens) (Fittkau, 1962). In Japan only one species of Nilotanypus has been recorded: Ni. minutus (Tokunaga), which is closely allied to dubius described by Tokunaga (1937). However, we could not find significant differences between dubius and minutus. Probably they are the same species.

\section{REFERENCES}

Brundin L, 1956. Zur Systematic der Orthocladiinae (Diptera: Chironomidae). Report Institute of Freshwater Research, Drottningholm, 37:5-185.

Cranston PS, Oliver DR, Saether OA, 1989. The adult males of Orthocladiinae (Diptera: Chironomidae) of the Holartic region. In: Chironomidae of the Holartic region: keys and diagnoses (Ed., Wiederholm T). Entomolgica Scandinavica Supplement, 34:245-246.

Edwards FW, 1924. Results of the Merton College Expedition to Spirtsbergen, 1923. IV. Diptera Newmatocera. Annals on Mag Natural History, 9:162-174.

Edwards FW, 1929. British non-biting midges (Diptera, Chironomidae). Transaction of the Royal Entomological Society of London, 77:279-430.

Fittkau EJ, 1962. Die Tanypodinae (Diptera, Chironomidae): die tribus Anatopinini, Macropelopiini und Pentaneurini. Akademie-Verlag, Berlin, pp. 412-413.

Goetghebuer M, 1932. Dipteres, Chironomidae. IV. Orthocladiinae, Corynoneurinae, Clunioninae, Diamesinae. Faune de France, 23:1-204. 
Goetghebuer M, 1937. Tendipedidae. B) Subfamilie Tendipedinae. In: Die Fliegen der Palaearktisheen Region (Ed., Lindner E). Hauptkonservator an der Wurtt, Naturaliensammlung, Stuttgart, pp.1-138.

Lee KY, Kang HY, Kim DS, Kim KE, Jeong BJ, Ree HI, 1995. Immunological responses to Chironomus flaviplumus in atopic children. Journal of Korean Society of Allergology, 15: 250-261.

Meigen JW, 1818. Systematische Beschreibung der bekannten europäischen zweiflügeligen Insekten. Erster Theil, Aachen, pp. 1-264.

Pinder LCV, 1978. A key to the adult males of British chironomidae (Diptera), the non-biting midges. Freshwater Biological Association Scientific Publication, 37:1-169.

Ree HI, Jeong KY, Nam SH, 2011. Six new and four unrecorded species of Tanytarsini (Diptera, Chironomidae, Chironominae) found in Korea. Korean Journal of Systematic Zoology, 27:246-261.

Ree HI, Jeong KY, Nam SH, Yong TS, 2010. Nine Polypedilum species (Diptera, Chironomidae) new to Korea collected near Namdae-stream, Muju. Korean Journal of Systematic Zoology, 26:203-216.

Ree HI, Kim HS, 1981. Studies on Chironomidae (Diptera) in Korea. I. Taxonomical study on adults of Chironomidae. Proceedings of the College of Natural Sciences, Seoul National University, 6:123-226.

Saether OA, 1980. Glossary of chironomid morphology terminology (Diptera: Chironomidae). Entomologica Scandinavica Supplement, 14:1-51.

Sasa M, 1981. Studies on chironomid midges of the Tama river.
Part 3. Species of the subfamily Orthocladiinae recorded at the summer survey and their distribution in relation to the pollution with sewage waters. Research Report on National Institute of Environmental Studies, 29:1-77.

Sasa M, 1990. On the chironomid midges of Jinzu river (Diptera, Chironomidae). Research Report of Toyama Prefectural Environmental Pollution Research Center, 1990:29-67.

Sasa M, Kawai K, 1987. Studies on the chironomid midges of Lake Biwa (Diptera, Chironomidae). Lake Biwa Study Monograph, 3:1-119.

Sasa M, Okazawa T, 1992. Studies on the chironomid midges (Yusurika) of Kurebe river. Research Report of Toyama Prefectural Environmental Pollution Research Center, 1992:4091.

Tokunaga M, 1937. Chironomidae from Japan (Diptera), IX Tanypodinae and Diamesinae. Philippine Journal of Science, 62:21-65.

Tokunaga M, 1939. Chironomidae from Japan. XI. New or little-known midges, with special reference to the metamorphosis of torrential species. Philiphine Journal of Science, 69:297-345.

Yong TS, Lee JS, Lee IY, Park SJ, Park GM, Ree HI, Park JW, Hong CS, Park HS, 1999. Identification of Chironomus kiiensis allergens, a dominant species of non-biting midges in Korea. Korean Journal of Parasitology, 37:171-179.

Zetterstedt JW, 1850. Chironomidae. Diptera Scandinaviae Disposita et Discripta, 9:3369-3710.

Received July 26, 2011 Revised December 15, 2011 Accepted December 20, 2011 